\title{
Essential (Cu, Zn) And Nonessential Metals (Pb, Cd) In Muscle of Leopard Groupers (Mycteroperca Rosacea) From A Mining Port In The Gulf of California, Mexico: Human Health Risk Assessment
}

\author{
Marina Soledad Irigoyen-Arredondo \\ Instituto Politécnico Nacional
}

Xchel Gabriel Moreno-Sánchez

Instituto Politécnico Nacional: Instituto Politecnico Nacional

Ofelia Escobar-Sánchez ( $\nabla$ escobars.ofelia@gmail.com)

Universidad Autónoma de Sinaloa https://orcid.org/0000-0002-7841-0080

Martín Federico Soto-Jiménez

ICMYL: Universidad Nacional Autonoma de Mexico Instituto de Ciencias del Mar y Limnologia

\section{Emigdio Marín-Enríquez}

Universidad Autónoma de Sinaloa: Universidad Autonoma de Sinaloa

\section{Leonardo Andrés Abitia-Cárdenas}

Instituto Politécnico Nacional: Instituto Politecnico Nacional

\section{Research Article}

Keywords: heavy metals, Epinephelidae, groupers, hazard quotient, health benefits, mining hotspot

Posted Date: August 4th, 2021

DOI: https://doi.org/10.21203/rs.3.rs-700350/v1

License: (c) (1) This work is licensed under a Creative Commons Attribution 4.0 International License.

Read Full License

Version of Record: A version of this preprint was published at Environmental Science and Pollution Research on January 19th, 2022. See the published version at https://doi.org/10.1007/s11356-02218753-7. 


\section{Abstract}

The concentrations of essential $(\mathrm{Cu}, \mathrm{Zn})$ and toxic $(\mathrm{Cd}, \mathrm{Pb})$ elements were quantified in the muscle of leopard groupers, Mycteroperca rosacea, in a mining district in the Gulf of California. Metal concentrations were analyzed by size, sex, maturity, season, year, and a risk factor for human consumption. Results indicated elevated levels of essential elements (Cu: $11 \pm 34.3 \mu \mathrm{g} / \mathrm{g}, \mathrm{Zn}: 377 \pm 1390$ $\mu \mathrm{g} / \mathrm{g}$ ), up to 10 times higher than concentrations of toxic elements (Cd: $0.06 \pm 0.1 \mu \mathrm{g} / \mathrm{g}, \mathrm{Pb}: 0.98 \pm 1.5$ $\mu \mathrm{g} / \mathrm{g})$. Cadmium was within permissible limit stated by Mexican norm $(0.5 \mu \mathrm{g} / \mathrm{g})$, but lead bordering its limit $(1.0 \mu \mathrm{g} / \mathrm{g})$. Heavy metal concentrations were comparable between males and females. Metal variations were not significantly correlated to the sex, maturity, season, or year $(p>0.05)$. The evaluation of benefits (Daily Mineral Intake) and risks (Target Hazard Quotients) to health indicated that these fish did not represent adverse effects to the consumer, within worldwide limits, but the nutritional benefits are high.

\section{Introduction}

The presence of heavy metals in marine ecosystems is one of the main contamination issues that leads to serious ecological and economic consequences (Ali and Khan 2019). Heavy metals are derived mainly from anthropogenic activities, such as dumping of residual waters and mining waste (Huerta-Díaz et al. 2014) which can contain persistent elements such as mercury ( $\mathrm{Hg})$, lead $(\mathrm{Pb})$, and cadmium (Cd) with non-known biological function but considered potentially toxic elements, at even low concentrations (Ali and Khan 2019). Anthropogenic inputs related to mining waste can also contain copper (Cu) and zinc (Zn), essential micronutrients for numerous physiological functions (Xu et al. 2017; Rehman et al. 2019). However, if permissible limits are surpassed, these essential elements could also be harmful to human health. It is therefore recommended the nutritional and toxicological analysis of organisms in the human diets to assess the potential to benefit or health risk that represent for the population, mainly for children, pregnant women, and lactating mothers.

Marine species fish are an important component in human diets worldwide, because they contain essential amino acids, fatty acids (especially omega-3 and omega-6), protein, vitamins, and minerals (Pal et al. 2018). Thus, recommendations for the human diet include increasing the consumption of fish, due to fish fillets to 2 to 4 times per week can reduce the risk of cardiovascular diseases, diabetes, and obesity (FAO 2017). By 2016, the global per capita consumption of white fish (low in fat, rich in proteins and minerals) accounts $20.5 \mathrm{~kg}$. White fish include fish species that represent approximately $90 \%$ of catches worldwide, such as tunas, snappers, and groupers (FAO 2017).

Many species of groupers are important commercial fish, and some of them are now used in other economic activities (e.g., aquaculture, sport fishing). In Mexico, the leopard grouper Mycteroperca rosacea (Streets 1877) is one of the most important species caught in the Gulf of California (Thomson et al. 2000). The annual production averaged 6,360 tons/year in 2014 and 2015, being the fourth species, in 
terms of catch volume, after sardines, shrimp, and sharks (CONAPESCA 2014). Its meat is categorized as high-quality, reaching high prices in local (\$7-10 USD per kilo) and national markets.

Ecologically, leopard groupers are among the main predators in coastal environments (Craig and Hastings 2007). They occupy a high trophic level ( 4.5) (Froese and Pauly 2021) with slow growth (from 15 to 21 years) (Díaz-Uribe et al. 2001), which makes them susceptible to accumulate high concentrations of heavy metals in tissues and organs. Overall, higher levels of heavy metals are usually associated with carnivorous and long-lived fish species (Evers et al. 2009). Despite its restricted distribution, high economic value, and scarce information on its biology, $M$. rosacea is no longer cataloged as "Vulnerable" by the International Union for the Conservation of Nature (IUCN) but currently cataloged as "Least concern" (Erisman and Craig 2018). Although non-changes in the leopard grouper populations have been reported over the past 30 years, enforcement in their population monitoring is required due to intense fishing pressure (Erisman and Craig 2018).

Fishing pressure on the leopard grouper occurs along the Gulf of California coastlines, however, their meat is local and nationally consumed. The objective of the present study was to evaluate the metal variability in function of fish biology (size, sex and maturity) and collection time (season and year). A second objective was to assess the potential human health benefit or risks related to concentrations of $\mathrm{Cu}, \mathrm{Pb}, \mathrm{Cd}$, and $\mathrm{Zn}$ in the muscle tissue of the leopard grouper $M$. rosacea. sharks

\section{Materials And Methods}

\section{Study area and sample collection}

Specimens of Mycteroperca rosacea were obtained in the port of Santa Rosalía ( $27^{\circ} 20.353$ ' N; $112^{\circ}$ 15.797' W), Baja California Sur, in the Gulf of California, Mexico (Fig. 1). Since 1885, this port has been extracting copper through the French mining company "Compagnie du Boleo, S.A.". Currently, the company operates under the name "Minera y Metalúrgica del Boleo S.A. de C.V." (Huerta-Díaz et al. 2014). About three million tons of accumulated slag are accumulated in the mine site, but unquantified amounts are already deposited on the adjacent marine environment (Shumilin et al. 2013; Huerta-Díaz et al. 2014). In addition to the mining industry, Santa Rosalía is height port for fishing and tourism vessels, and cargo and passenger ferries, and numerous small boats (Huerta-Díaz et al. 2014). The marine environment is, therefore, potentially polluted for heavy metals and marine organisms that inhabit the area, including the leopard grouper, being impacted.

Organisms were captured monthly (March 2014 to May 2015) by spearfishing while free diving, trying to obtain a good representation of different sizes. Specimens were stored on ice until processing at the Fish Ecology Laboratory at CICIMAR-IPN, where the total length $(\mathrm{TL}, \mathrm{cm})$ and weight $(\mathrm{g})$ were recorded; sex was identified by direct observation of the gonads and later corroborated with histological analyses (Nikolsky 1963; Pérez-Olivas et al. 2018). Four maturity stages were considered according to Pérez-Olivas et al. (2018). 
A total of $345 \mathrm{M}$. rosacea individuals was caught, with sizes ranging from 21 to $74 \mathrm{~cm}$ total length; 93 specimens were male and 185 were female. A total of 15 bisexual immature organisms, as well as, 52 individuals for which sex could not be histologically identified. These specimens were excluded in the comparative analyses by sex and maturity stages. According to fish size, the specimens were binned in small ( $n=161,47 \%)$, medium $(n=148,43 \%)$, and large $(n=36,10 \%)$. Besides, the specimens were categorized by caught season, in cold $(n=244,71 \%)$ and warm season $(n=101,21 \%)$, by year: $2014(n=$ $180,72 \%)$ and $2015(n=68,27 \%)$.

Fish were dissected and the skin was removed to extract $5.0 \mathrm{~g}$ of muscle tissue from the anterior dorsal part of each individual. Each sample was tagged, stored in plastic bags, and frozen at $-20^{\circ} \mathrm{C}$.

\section{Heavy metal analysis}

Muscle tissues samples were lyophilize at a $0.120 \mathrm{mBar}$ pressure and $-40^{\circ} \mathrm{C}$ for $72 \mathrm{~h}$ (Labconco, FreeZone 2.5). The water content (\%) was calculated by weigh differences between fresh frozen and dried sample. The dried samples were ground using an agate mortar, homogenized, and packed into trace metal cleaned plastic vials.

Then, a lot of 248 composite muscle samples were sent to the Stable Isotope Laboratory at the ICMYLUNAM at Mazatlán for the elemental analysis. Samples were processed and analyzed in HEPA filtered air (Class 1000), trace metal clean laboratory using high-purity reagents (trace metal grade) and water (18 $\mathrm{MW} \mathrm{cm}{ }^{-1}$; Milli-Q academic). Aliquots of fish muscle samples, blanks and certified reference material were digested in Teflon vials (Savillex) with $10 \mathrm{~mL}$ concentrated nitric acid $\left(\mathrm{HNO}_{3}\right)$. Containers were then placed on mod-block unit $\left(120^{\circ} \mathrm{C}\right)$ for $4 \mathrm{~h}$. After digestion, samples were transferred to polyethylene vials and diluted with Milli-Q water to known volume (about $25 \mathrm{~mL}$ ).

Elements were analyzed in a Thermo Scientific Element XR magnetic sector high-resolution inductively coupled plasma source mass spectrometry (HR-ICP-MS) (Soto-Jiménez et al. 2008). Accuracy of the analyses was determined with concurrent processing and analysis of certified reference material (CRM) Dog-Fish muscle (DORM-3) of Institute for National Measurement Standards of the National Research Council Canada. Recovery values of CRM for Cd was $92 \%$, Cu $94 \%$, Pb $88 \%$, and $\mathrm{Zn} 92 \%$. The detection limits were $<6 \mathrm{ng} \mathrm{g}^{-1}$ dry weight for $\mathrm{Cd},<10 \mathrm{ng} \mathrm{g}^{-1}$ for $\mathrm{Pb}$, and $<20 \mathrm{ng} \mathrm{g}^{-1}$ for $\mathrm{Cu}$ and $\mathrm{Zn}$. Concentrations of metals in fish were calculated in $\mu \mathrm{g} / \mathrm{g}$ dry weight but reported as averages \pm standard deviations in $\mu \mathrm{g} / \mathrm{g}$ wet weight.

\section{Toxicological evaluation}

The provisional tolerable weekly intake (PTWI) of Cu, Cd, Pb, and Zn (PTWI, in $\mu$ g week ${ }^{-1} \mathrm{~kg}^{-1}$ of body weight) to establish the amount of leopard grouper fillet that the human population could consume without a health risk, was calculated by PTWI $=[F A W][M T][P A W]$. 
Where FAW is the average weight of fish fillet consumed by an adult (12 kg per person per year; De la 0Burrola et al. 2015), MT is the metal concentration ( $\mu \mathrm{g} / \mathrm{g}, \mathrm{w} . \mathrm{w}$.) in muscle tissue of leopard grouper, and PAW is the average weight of the general population, considering women (pregnant and lactating) and children (4 to 6 years old).

Average worldwide weights established by the World Health Organization (e.g., $70 \mathrm{~kg}$ for men, $60 \mathrm{~kg}$ for women, and $16 \mathrm{~kg}$ for 4-6 year old children) and the average weights for the Santa Rosalía population (75 kg for men, 69 kg for women, and 18 kg for 4-6 year old children) (INEGI 2015) were taken into account. These average weights were also used for further evaluations.

The daily mineral intake of essential elements $(\mathrm{Cu}, \mathrm{Zn})$ was also calculated based on the consumption recommended by the Institute of Medicine of the National Academies of the United States (www.iom.edu), using the following equation: $\mathrm{DMI}=\mathrm{C}$ * $100 / \mathrm{RDI}$

Where $\mathrm{DMI}=$ daily mineral intake of essential elements, $\mathrm{C}=\mathrm{Cu}$ or Zn concentration in $100 \mathrm{~g}$ of fresh fish weight, $\mathrm{RDI}$ = recommended daily intake, estimated as safe and adequate for each group in the population. CuRDI: men, women, and children $=900 \mu \mathrm{g} /$ day, pregnant and lactating women $=1000 \mu \mathrm{g}$ /day; ZnRDI: men, women, pregnant, and lactating women = $40 \mu \mathrm{g} / \mathrm{day}$, children $=34 \mu \mathrm{g} / \mathrm{day}$. These values were estimated for a healthy population.

Additionally, the potential health risk due to harmful effects from long-term leopard grouper consumption (e.g., months or years) was calculated as the target hazard quotient, where high THQ values (>1) would represent a health risk. The THQ was calculated as follows:

$T H Q=\frac{E F * E D * F I R * C}{R f D * B W * A T}$

Where $\mathrm{C}$ is the concentration of $\mathrm{Cu}, \mathrm{Pb}, \mathrm{Cd}$, or $\mathrm{Zn}(\mu \mathrm{g} / \mathrm{g})$; EF is the exposure frequency (days/year); ED is the exposure duration (years/time); FIR is the fish intake rate (g/day); RfD is the daily fish intake rate (g/day); BW is the average body weight of the human population (kg); and AT is the average exposure time (days). All parameters except C, FIR, and BW were obtained from real data for the Santa Rosalía, BCS, Mexico population. The remaining data used in the formula were obtained from the FAO (2017), US EPA (2015), and Yi et al. (2011) and are shown in Table 1.

Table 1 Parameters and values used for the THQ formula for each heavy metal 


\begin{tabular}{|lllll|}
\hline Factor & Definition & Unit & Value & Reference \\
\hline EF & $\begin{array}{l}\text { Exposure } \\
\text { frequency }\end{array}$ & Days/year & 365 & $\begin{array}{l}\text { Yi et al. } \\
(2011)\end{array}$ \\
\hline ED & Duration & Years & 78 & $\begin{array}{l}\text { Yi et al. } \\
(2011)\end{array}$ \\
\hline FIR & Fish intake rate & $\mathrm{g} /$ day & 32.8 & \\
\hline RfD & Reference dose & $\mu \mathrm{g} / \mathrm{kg} /$ day & $1.0 \mathrm{E}^{-01}(\mathrm{Cd}), 2.0 \mathrm{E}^{+01}(\mathrm{~Pb}), 4.0 \mathrm{E}^{+01}(\mathrm{Cu})$, & $\begin{array}{l}\text { US EPA } \\
(2015)\end{array}$ \\
\hline BW & Average weight & $\mathrm{Kg}$ & $70 \pm 75$ & $\begin{array}{l}\text { WHO; INEGI } \\
(2015)\end{array}$ \\
\hline AT & $\begin{array}{llll}\text { Average exposure } \\
\text { time }\end{array}$ & Days & $365^{*} 78=28.470$ & $\begin{array}{l}\text { Yi et al. } \\
(2011)\end{array}$ \\
\hline
\end{tabular}

Because heavy metal ( $\mathrm{Cu}, \mathrm{Pb}, \mathrm{Cd}$, and $\mathrm{Zn}$ ) interactions can cause multiple effects (Gu et al., 2017), we considered the added effect of these elements in the THQ as was proposed by Chien et al. (2002) and Gu et al. (2017), as shown in the following equation:

Total THQ $(T T H Q)=\sum T H Q_{C u, P b, C d, Z n}$

\section{Data analysis}

Data were grouped by sex (males and females), maturity stages (stage 1, stage 2, stage 3 and stage 4) and size (small: $<36 \mathrm{~cm}$, medium: $>36 \mathrm{~cm}$ and $\leq 51 \mathrm{~cm}$, large: $>51 \mathrm{~cm}$ ). Seasonality was defined according to temperature records for the study period obtained from MODIS-AQUA satellite images with $1.1 \mathrm{~km}$ resolution. Anomalies were recorded based on the annual average of $23^{\circ} \mathrm{C}$ (Moreno-Sánchez et al. 2019). Months with positive anomalies were assigned to the warm season and negative anomalies were assigned to the cold season. Simple linear regression (LR) analysis was carried to assess the association between the concentration of each heavy metal and the size of leopard groupers. Data base was split into categories (sex, maturity stages and season) and a LR of the heavy metal concentration as a function of size was fitted for each heavy metal and each category. With this analysis, the hypothesis that the $b$ coefficient of the LR model was zero (e.g. no association between heavy metal concentration and size) was tested. All analyses were performed using packages found in R (R Core Team 2017).

\section{Results}

\section{Heavy metal concentrations in muscle tissue}

A summary of the heavy metal concentrations in muscle of leopard grouper M. rosacea caught in Santa Rosalía (average $\pm S D$, maximum, and minimum), and the analysis comparative for sex, maturity stages, total length, and season in provided in Table 2. In agreement to the sex groups, males (Cu: $13.64 \pm 33.64$ 
$\mu \mathrm{g} / \mathrm{g}$; Zn: $409.68 \pm 1348.08 \mu \mathrm{g} / \mathrm{g}$; Cd: $0.08 \pm 0.20 \mu \mathrm{g} / \mathrm{g} ; \mathrm{Pb}: 0.84 \pm 1.0 \mu \mathrm{g} / \mathrm{g}$ ), females (Cu: $12.77 \pm 39.93$ $\mu \mathrm{g} / \mathrm{g} ; \mathrm{Zn}: 417.40 \pm 1628.53 \mu \mathrm{g} / \mathrm{g}$; Cd: $0.05 \pm 0.12 \mu \mathrm{g} / \mathrm{g}: \mathrm{Pb}: 1.06 \pm 1.7 \mu \mathrm{g} / \mathrm{g}$ ) and hermaphrodites (Cu: 7.74 $\pm 7.75 \mu \mathrm{g} / \mathrm{g} ; \mathrm{Zn}: 164.75 \pm 219.94 \mu \mathrm{g} / \mathrm{g} ; \mathrm{Cd}: 0.07 \pm 0.10 \mu \mathrm{g} / \mathrm{g} ; \mathrm{Pb}: 0.79 \mathrm{~b} \pm 1.3 \mu \mathrm{g} / \mathrm{g}$ ) showed comparable ranges for all metals. Because the comparable values and the high intravariability among sex groups, non-significant differences were observed. 
Table 2

Heavy metal concentrations (mean $\pm \mathrm{SD} ; \mu \mathrm{g} / \mathrm{g}$ wet weight) in Mycteroperca rosacea muscle in a mining port in the Gulf of California, Mexico

\begin{tabular}{|c|c|c|c|c|c|}
\hline Category & $n$ & $\mathrm{Cu}$ & $\mathrm{Zn}$ & $\mathrm{Cd}$ & $\mathrm{Pb}$ \\
\hline Overall & 248 & $\begin{array}{l}11.6 \pm 34.3 \\
(0.01- \\
361)^{c}\end{array}$ & $\begin{array}{l}377.3 \pm 1389.9 \\
(0.02-13165)^{c}\end{array}$ & $\begin{array}{l}0.06 \pm 0.13 \\
(0.001- \\
1.13)\end{array}$ & $\begin{array}{l}0.98 \pm 1.56 \\
(0.03-9.8)^{b}\end{array}$ \\
\hline
\end{tabular}

Sex

\begin{tabular}{|c|c|c|c|c|c|}
\hline Females & 132 & $\begin{array}{l}12.7 \pm 39.9 \\
(0.01- \\
361)^{c}\end{array}$ & $\begin{array}{l}433.8 \pm 1628.5 \\
(0.02-13165)^{\mathrm{C}}\end{array}$ & $\begin{array}{l}0.05 \pm 0.12 \\
(0.001- \\
0.69)\end{array}$ & $\begin{array}{l}1.06 \pm 1.71 \\
(0.03- \\
8.9)^{a, b}\end{array}$ \\
\hline Males & 70 & $\begin{array}{l}13.6 \pm 33.6 \\
(0.1-226)^{\mathrm{c}}\end{array}$ & $\begin{array}{l}448.8 \pm 1348 \\
(0.01-9442)^{c}\end{array}$ & $\begin{array}{l}0.08 \pm 0.2 \\
(0.01-1.1)\end{array}$ & $\begin{array}{l}0.84 \pm 1.0 \\
(0.02-9.8)^{b}\end{array}$ \\
\hline erm & 15 & $\begin{array}{l}7.7 \pm 7.7 \\
(0.02-20)^{c}\end{array}$ & $\begin{array}{l}164.7 \pm 219.9 \\
(0.1-807)^{\mathrm{c}}\end{array}$ & $\begin{array}{l}0.07 \pm 0.1 \\
(0.001-0.3)\end{array}$ & $\begin{array}{l}0.79 \pm 1.3 \\
(0.02-5.3)^{b}\end{array}$ \\
\hline
\end{tabular}

Size

$\begin{array}{llllll}\text { Small } & 129 & \begin{array}{l}12 \pm 34 \\ (0.2-361)^{\mathrm{c}}\end{array} & \begin{array}{l}385 \pm 1644 \\ (0.1-13165)^{\mathrm{c}}\end{array} & \begin{array}{l}0.06 \pm 0.1 \\ (0.01-1.1)\end{array} & \begin{array}{l}0.98 \pm 1.6 \\ (0.1-9.8)^{\mathrm{b}}\end{array} \\ \text { Medium } & 99 & \begin{array}{l}11 \pm 32 \\ (0.01-\end{array} & \begin{array}{l}328 \pm 1269 \\ (0.02-13165)^{\mathrm{c}}\end{array} & \begin{array}{l}0.06 \pm 0.1 \\ (0.01-0.7)\end{array} & \begin{array}{l}1.0 \pm 1.5 \\ (0.02-9.8)^{\mathrm{a}} \\ \mathrm{b}\end{array} \\ & & 361)^{\mathrm{c}} & & & \\ \text { Large } & 20 & \begin{array}{l}7 \pm 32(0.2- \\ 90)^{\mathrm{c}}\end{array} & \begin{array}{l}246 \pm 1330 \\ (0.8-6901)^{\mathrm{c}}\end{array} & \begin{array}{l}0.03 \pm 0.07 \\ (0.01-0.1)\end{array} & \begin{array}{l}0.86 \pm 1.4 \\ (0.05-2.7)^{\mathrm{b}}\end{array}\end{array}$

Maturity

stage

\begin{tabular}{|c|c|c|c|c|c|}
\hline $\begin{array}{l}\text { Maturity stage } \\
1\end{array}$ & 141 & $\begin{array}{l}18.1 \pm 58.4 \\
(0.3-283)^{c}\end{array}$ & $\begin{array}{l}588 \pm 2394 \\
(2.3-12006)^{c}\end{array}$ & $\begin{array}{l}0.1 \pm 0.2 \\
(0.01- \\
0.8)^{a, b}\end{array}$ & $\begin{array}{l}1.5 \pm 2.4 \\
(0.07- \\
15.4)^{a, b}\end{array}$ \\
\hline $\begin{array}{l}\text { Maturity stage } \\
2\end{array}$ & 47 & $\begin{array}{l}16.8 \pm 55.6 \\
(0.3-123)^{\mathrm{c}}\end{array}$ & $\begin{array}{l}517.9 \pm 2256 \\
(6.0-5767)^{\mathrm{c}}\end{array}$ & $\begin{array}{l}0.1 \pm 0.2 \\
(0.01- \\
1.3)^{\mathrm{a}, \mathrm{b}}\end{array}$ & $\begin{array}{l}1.5 \pm 2.4 \\
(0.06- \\
11.0)^{a, b}\end{array}$ \\
\hline $\begin{array}{l}\text { Maturity stage } \\
3\end{array}$ & 76 & $\begin{array}{l}13.8 \pm 56.7 \\
(0.3-664)^{\mathrm{c}}\end{array}$ & $\begin{array}{l}430.2 \pm 2323 \\
(3.7-24197)^{c}\end{array}$ & $\begin{array}{l}0.1 \pm 0.2 \\
(0.01- \\
0.9)^{\mathrm{a}, \mathrm{b}}\end{array}$ & $\begin{array}{l}1.4 \pm 2.3 \\
(0.06- \\
6.5)^{a, b}\end{array}$ \\
\hline
\end{tabular}

a Values above recommended limits set by the official Mexican norm NOM-242-SSA1-2009 (Cd 0.5

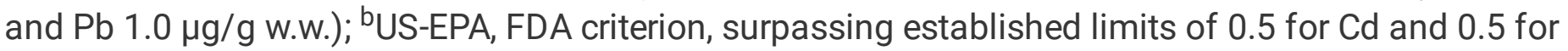
$\mathrm{Pb}$; 'United Kingdom norm (UK-EEA food standards) of Zn 50 and $\mathrm{Cu} 5$ gg/g w.w. 


\begin{tabular}{|c|c|c|c|c|c|c|}
\hline Category & & $n$ & $\mathrm{Cu}$ & $\mathrm{Zn}$ & Cd & $\mathrm{Pb}$ \\
\hline & $\begin{array}{l}\text { Maturity stage } \\
4\end{array}$ & 8 & $\begin{array}{l}14.1 \pm 58.1 \\
(0.6-132)^{c}\end{array}$ & $\begin{array}{l}447.3 \pm 2362 \\
(12.2-2231)^{c}\end{array}$ & $\begin{array}{l}0.1 \pm 0.2 \\
(0.02- \\
0.1)^{a, b}\end{array}$ & $\begin{array}{l}1.6 \pm 2.5 \\
(0.05- \\
1.7)^{a, b}\end{array}$ \\
\hline \multicolumn{7}{|l|}{ Season } \\
\hline & Warm season & 80 & $\begin{array}{l}2.0 \pm 3.0 \\
(0.2-23)\end{array}$ & $\begin{array}{l}50 \pm 66(0.8- \\
411)^{c}\end{array}$ & $\begin{array}{l}0.01 \pm 0.03 \\
(0.02-0.11)\end{array}$ & $\begin{array}{l}1.0 \pm 2.0 \\
(0.05- \\
9.8)^{a, b}\end{array}$ \\
\hline & Cold season & 168 & $\begin{array}{l}16 \pm 35 \\
(0.1-361)^{c}\end{array}$ & $\begin{array}{l}539 \pm 1412 \\
(0.1-13165)^{c}\end{array}$ & $\begin{array}{l}0.11 \pm 0.15 \\
(0.01- \\
1.1)^{\mathrm{a}, \mathrm{b}}\end{array}$ & $\begin{array}{l}1.0 \pm 1.6 \\
(0.02- \\
8.9)^{a, b}\end{array}$ \\
\hline \multicolumn{7}{|l|}{ Year } \\
\hline & 2014 & 180 & $\begin{array}{l}8.8 \pm 31.5 \\
(0.17- \\
361)^{c}\end{array}$ & $\begin{array}{l}251 \pm 1239 \\
(0.1-13165)^{c}\end{array}$ & $\begin{array}{l}0.08 \pm 0.2 \\
(0.02-1.1)\end{array}$ & $\begin{array}{l}0.97 \pm 1.6 \\
(0.03-9.9)^{\mathrm{b}}\end{array}$ \\
\hline & 2015 & 68 & $\begin{array}{l}26 \pm 38.4 \\
(0.2-205)^{\mathrm{c}}\end{array}$ & $\begin{array}{l}867 \pm 1499.7 \\
(5.1-8702)^{c}\end{array}$ & $\begin{array}{l}0.2 \pm 0.3 \\
(0.1-1.1)^{b}\end{array}$ & $\begin{array}{l}1.5 \pm 1.9 \\
(0.1-1.9)^{\mathrm{a}, \mathrm{b}}\end{array}$ \\
\hline \multicolumn{7}{|c|}{$\begin{array}{l}\text { aValues above recommended limits set by the official Mexican norm NOM-242-SSA1-2009 (Cd } 0.5 \\
\text { and Pb } 1.0 \mu \mathrm{g} / \mathrm{g} \text { w.w.); bUS-EPA, FDA criterion, surpassing established limits of } 0.5 \text { for Cd and } 0.5 \text { for } \\
\mathrm{Pb} \text {; CUnited Kingdom norm (UK-EEA food standards) of Zn } 50 \text { and Cu } 5 \mu \mathrm{g} / \mathrm{g} \text { w.w. }\end{array}$} \\
\hline
\end{tabular}

Heavy metal concentrations in maturity stages were, stage 1 (Cu: $18.1 \pm 58.4 \mu \mathrm{g} / \mathrm{g} ; \mathrm{Zn}: 588 \pm 2394 \mu \mathrm{g} / \mathrm{g}$; Cd: $0.1 \pm 0.2 \mu \mathrm{g} / \mathrm{g} ; \mathrm{Pb}: 1.5 \pm 2.4 \mu \mathrm{g} / \mathrm{g}$ ), stage 2 (Cu: $16.8 \pm 55.6 \mu \mathrm{g} / \mathrm{g} ; \mathrm{Zn}: 517.9 \pm 2256 \mu \mathrm{g} / \mathrm{g} ; \mathrm{Cd}: 0.1 \pm 0.2$ $\mu \mathrm{g} / \mathrm{g} ; \mathrm{Pb}: 1.5 \pm 2.4 \mu \mathrm{g} / \mathrm{g}$ ), stage 3 (Cu: $13.8 \pm 56.7 \mu \mathrm{g} / \mathrm{g} ; \mathrm{Zn}: 439.2 \pm 2323 \mu \mathrm{g} / \mathrm{g} ; \mathrm{Cd}: 0.1 \pm 0.2 \mu \mathrm{g} / \mathrm{g} ; \mathrm{Pb}: 1.4$ $\pm 2.3 \mu \mathrm{g} / \mathrm{g}$ ) and stage 4 (Cu: $14.1 \pm 58.1 \mu \mathrm{g} / \mathrm{g} ; \mathrm{Zn}: 447.3 \pm 2362 \mu \mathrm{g} / \mathrm{g} ; \mathrm{Cd}: 0.1 \pm 0.2 \mu \mathrm{g} / \mathrm{g} ; \mathrm{Pb}: 1.6 \pm 2.5$ $\mu \mathrm{g} / \mathrm{g})$. There were non-significant differences in maturity stages between metals levels.

Regarding size, the metal levels were orders as follow, small fish (Cu: $12 \pm 34 \mu \mathrm{g} / \mathrm{g} ; \mathrm{Zn}: 385 \pm 1644 \mu \mathrm{g} / \mathrm{g}$; Cd: $0.06 \pm 0.1 \mu \mathrm{g} / \mathrm{g} ; \mathrm{Pb}: 0.98 \pm 1.6 \mu \mathrm{g} / \mathrm{g}$ ), then medium-sized fish (Cu:11 $\pm 32 \mu \mathrm{g} / \mathrm{g} ; Z n: 328 \pm 1269 \mu \mathrm{g} / \mathrm{g}$; Cd: $0.06 \pm 0.1 \mu \mathrm{g} / \mathrm{g} ; \mathrm{Pb}: 1.0 \pm 1.5 \mu \mathrm{g} / \mathrm{g}$ ) and large specimens presented lower concentrations (Cu:7.0 \pm 32 $\mu \mathrm{g} / \mathrm{g} ; \mathrm{Zn}: 246 \pm 1330 \mu \mathrm{g} / \mathrm{g} ; \mathrm{Cd}: 0.03 \pm 0.07 \mu \mathrm{g} / \mathrm{g} ; \mathrm{Pb}: 0.86 \pm 1.4 \mu \mathrm{g} / \mathrm{g})$. Non-significant differences were observed among size groups.

An analysis comparative related to the collection time, showed that the heavy metal concentrations in organisms collected in 2015 (Cu: $26 \pm 38.4 \mu \mathrm{g} / \mathrm{g}$; Zn: $867 \pm 1499 \mu \mathrm{g} / \mathrm{g}$; Cd: $0.2 \pm 0.3 ; \mathrm{Pb}: 1.5 \pm 1.9 \mu \mathrm{g} / \mathrm{g}$ ) were significantly higher $(p<0.05)$ than 2014 specimens (Cu: $8.8 \pm 31.5 \mu \mathrm{g} / \mathrm{g} ; \mathrm{Zn}: 251 \pm 1239 \mu \mathrm{g} / \mathrm{g}$; Cd: $0.08 \pm 0.2 \mu \mathrm{g} / \mathrm{g} ; \mathrm{Pb}: 0.97 \pm 1.6 \mu \mathrm{g} / \mathrm{g})$. Besides, significant differences $(\mathrm{p}<0.05)$ were observed for $\mathrm{Cu}, \mathrm{Zn}$ and Cd between warm (Cu: $2.0 \pm 3.0 \mu \mathrm{g} / \mathrm{g} ; \mathrm{Zn}: 50 \pm 66 \mu \mathrm{g} / \mathrm{g} ; \mathrm{Cd}: 0.01 \pm 0.03 \mu \mathrm{g} / \mathrm{g}$ ) and cold season (Cu: $16 \pm 35 \mu \mathrm{g} / \mathrm{g} ; Z \mathrm{Zn}: 539 \pm 1412 \mu \mathrm{g} / \mathrm{g} ;$ Cd: $0.11 \pm 0.15 \mu \mathrm{g} / \mathrm{g}$ ), but not for lead (warm: $1.0 \pm 2.0 \mu \mathrm{g} / \mathrm{g}$; cold: 1.0 $\pm 1.6 \mu \mathrm{g} / \mathrm{g})$. 
Results of the fitted simple linear regression (LR) suggested that the association between heavy metal concentration in muscle and size of leopard groupers is weak (low $R^{2}$ values) and non significant $(p(b=$ 0 ) $>0.05$ in all cases) (Table 3 ). 
Table 3

Coefficient values of the linear regression of variations by sex, maturity stages and season in the muscle of leopard grouper Mycteroperca rosacea in Santa Rosalía, BCS, Mexico

\begin{tabular}{|c|c|c|c|c|c|c|}
\hline \multirow[t]{2}{*}{ Variables } & \multicolumn{6}{|c|}{ Linear regression coefficients } \\
\hline & $n$ & $\mathrm{R}^{2}$ & b & a & $p(a=0)$ & $p(b=0)$ \\
\hline \multicolumn{7}{|l|}{$\mathrm{Cu}$} \\
\hline $\mathrm{F}$ & 132 & 0.004 & 0.3 & 2047030 & 1 & 0.46 \\
\hline$M$ & 69 & 0.01 & 0.4 & -2975734 & 0.87 & 0.36 \\
\hline Maturity stage 1 & 141 & 0.01 & 0.5 & -1018392 & 0.97 & 0.45 \\
\hline Maturity stage 2 & 47 & 0.02 & -0.48 & 26628406 & 0.02 & 0.14 \\
\hline Maturity stage 3 & 76 & 0.01 & 0.6 & -11830348 & 0.74 & 0.43 \\
\hline Maturity stage 4 & 8 & 0.14 & 2688 & -76258327 & 0.48 & 0.40 \\
\hline Cold & 148 & 0.01 & 0.5 & -2897376 & 0.85 & 0.19 \\
\hline Warm & 47 & 0.05 & -0.1 & 6542092 & 0.01 & 0.12 \\
\hline \multicolumn{7}{|l|}{$\mathrm{Pb}$} \\
\hline $\mathrm{F}$ & 132 & 0.0005 & -0.005 & 13403743 & 0.07 & 0.79 \\
\hline M & 69 & 0.04 & 0.02 & -0.1501317 & 0.75 & 0.07 \\
\hline Maturity stage 1 & 141 & 0.007 & -0.02 & 19128.434 & 0.03 & 0.39 \\
\hline Maturity stage 2 & 47 & 0.105 & 0.07 & -18344799 & 0.24 & 0.06 \\
\hline Maturity stage 3 & 76 & 0.04 & 0.02 & -0.1766029 & 0.77 & 0.13 \\
\hline Maturity stage 4 & 8 & 0.11 & 0.03 & -0.7844367 & 0.62 & 0.45 \\
\hline Cold & 148 & 0.0004 & -0.003 & 11716741 & 0.03 & 0.80 \\
\hline Warm & 47 & 0.01 & 0.02 & -0.1538668 & 0.90 & 0.42 \\
\hline \multicolumn{7}{|l|}{$\mathrm{Cd}$} \\
\hline $\mathrm{F}$ & 132 & 0.02 & -0.002 & 0.138755835 & 0.002 & 0.06 \\
\hline M & 69 & 0.005 & 0.001 & 0.010781516 & 0.92 & 0.54 \\
\hline Maturity stage 1 & 141 & 0.03 & -0.002 & 0.158315518 & 0.002 & 0.05 \\
\hline Maturity stage 2 & 47 & 0.001 & 0.001 & 0.088152186 & 0.74 & 0.83 \\
\hline Maturity stage 3 & 76 & 0.003 & 0.0005 & 0.007955513 & 0.89 & 0.70 \\
\hline Maturity stage 4 & 8 & 0.14 & 0.002 & -0.077131024 & 0.49 & 0.40 \\
\hline
\end{tabular}




\begin{tabular}{|lllllll|}
\hline Variables & \multicolumn{7}{l}{ Linear regression coefficients } & & \\
\cline { 2 - 7 } & $\mathbf{n}$ & $\mathbf{R}^{2}$ & $\mathbf{b}$ & $\mathbf{a}$ & $\mathbf{p}(\mathbf{a}=\mathbf{0})$ & $\mathbf{p}(\mathbf{b}=\mathbf{0})$ \\
\hline Cold & 148 & 0.001 & -0.0006 & 0.100902619 & 0.10 & 0.69 \\
\hline Warm & 47 & 0.02 & -0.0005 & 0.044550624 & 0.02 & 0.27 \\
\hline Zn & & & & & & \\
\hline F & 132 & 0.01 & 252802895 & -48738201 & 0.43 & 0.13 \\
\hline M & 69 & 0.009 & 151811566 & -15627183 & 0.83 & 0.42 \\
\hline Maturity stage 1 & 141 & 0.008 & -127235772 & 74047734 & 0.12 & 0.35 \\
\hline Maturity stage 2 & 47 & 0.005 & 87707420 & 9110244 & 0.91 & 0.67 \\
\hline Maturity stage 3 & 76 & 0.01 & 302079514 & -62109127 & 0.65 & 0.37 \\
\hline Maturity stage 4 & 8 & 0.13 & 453892443 & -129630397 & 0.48 & 0.41 \\
\hline Cold & 148 & 0.02 & 297236154 & -53587711 & 0.38 & 0.07 \\
\hline Warm & 47 & 0.02 & 0.69 & 1298858 & 0.62 & 0.31 \\
\hline
\end{tabular}

A comparison of metal concentrations found in the grouper muscle samples, evidenced that the essential elements $(\mathrm{Cu}=11.61 \pm 34.36 \mu \mathrm{g} / \mathrm{g}$ and $\mathrm{Zn}=377.33 \pm 1389.98 \mu \mathrm{g} / \mathrm{g})$ showed global values that exceeded the norms set by the United Kingdom (UK-EEA food standards Cu: $5.0 \mu \mathrm{g} / \mathrm{g} ; \mathrm{Zn}: 50 \mu \mathrm{g} / \mathrm{g}$ ), whereas Cd, a non-essential element $(C d=0.06 \pm 0.13 \mu \mathrm{g} / \mathrm{g}$ vs $\mathrm{Cd}=0.05 \mu \mathrm{g} / \mathrm{g})$ were within ranges established by NOM242 (Table 2). In the case of $\mathrm{Pb}$, the global average is bordering the limit established by NOM-242. Even, a numerous number of samples $(n=114,46 \%)$ are above the limit of $1 \mu \mathrm{g} / \mathrm{g}$.

\section{Ecotoxicological evaluation}

According to the Calculations of the maximum weekly intake values (MWIV) for Cd values, we suggest that children under 6 years can consume fish fillets of up to approximately $533 \mathrm{~g}$ per week and that women and men can consume up to $2,000 \mathrm{~g}$ of leopard grouper. However, $\mathrm{Pb}$ values indicated that the frequency of leopard grouper consumption should decrease. The average values of the risk coefficient $\left(\mathrm{THQ}_{\mathrm{Cu}}: 0.1 \pm 0.3, \mathrm{THQ}_{\mathrm{Pb}}: 0 \pm 0.1, \mathrm{THQ}_{\mathrm{Cd}}: 0.3 \pm 1.4, \mathrm{THQ}_{\mathrm{Zn}}: 0.2 \pm 0.6\right)$ showed there was no risk from leopard grouper ingestion; however, the maximum range of $\mathrm{THQ}_{\mathrm{Cd}}$ values could surpass established limits (>1). In contrast, the suggested amounts of essential elements ( $\mathrm{Cu}$ and $\mathrm{Zn}$ ) were higher for the three human population groups (children, women, and men).

The benefits that consumption of this fish provide for vulnerable groups in the population (children, pregnant, and lactating women) indicated that each $100 \mathrm{~g}$ of leopard grouper filet represented over $1 \%$ of $\mathrm{Cu}$ and $100 \%$ of $\mathrm{Zn}$. The percentages of Cu daily mineral intake oscillated between 1.2 and 1.3, while the percentages of $\mathrm{Zn}$ daily mineral intake ranged from 943 to 1,110 . 


\section{Discussion}

The leopard grouper Mycteroperca rosacea is one of the main predatory species on rocky reefs (Thomson et al. 2000; Moreno-Sánchez et al. 2019). Due to its carnivorous habits and slow growth (Díaz-Uribe et al. 2001), it can be susceptible to accumulating high levels of heavy metals, as occurs with other species with these characteristics, such as Scomberomorus sierra, Nematistius pectoralis, Caulolatilus princeps, and Lutjanus colorado (Frías-Espericueta et al. 2010; Ruelas-Inzunza et al. 2010, 2014). However, Cd concentrations $(0.06 \mu \mathrm{g} / \mathrm{g})$ were below the limit set by the Mexican norm NOM-242, whereas Pb concentrations found in $M$. rosacea muscle tissue $(0.98 \mu \mathrm{g} / \mathrm{g})$ were on average close to the limit set by the Mexican norm ( $\mathrm{Pb}: 1.0 \mu \mathrm{g} / \mathrm{g}$; Cd: $0.5 \mu \mathrm{g} / \mathrm{g}$ ). The average values of essential metals ( $\mathrm{Cu}: 11.61 \pm 34.36$ $\mu \mathrm{g} / \mathrm{g}$; Zn: $377.33 \pm 1389.98 \mu \mathrm{g} / \mathrm{g}$ ) were well above the maximum criteria allowed by international regulations (UK-EEA food standards Cu: $5.0 \mu \mathrm{g} / \mathrm{g}$; Zn: $50 \mu \mathrm{g} / \mathrm{g}$ ).

Levels of $\mathrm{Cd}(0.2 \mu \mathrm{g} / \mathrm{g})$ and $\mathrm{Pb}(2.5 \mu \mathrm{g} / \mathrm{g})$ one to three times higher than those recorded here for $M$. rosacea have been recorded in other commercially important species in Sinaloa, Mexico (S. sierra, $C$. princeps, and L. colorado). Coastal ecosystems in Sinaloa has a significant anthropogenic influence, raw or partially process effluents of aquaculture, agriculture, food processing industry, urban wastewater and fisheries converge (Frías-Espericueta et al. 2010; Ruelas-Inzunza et al. 2014). Concentrations of $\mathrm{Cu}, \mathrm{Zn}$, $\mathrm{Pb}$, and $\mathrm{Cd}$ above the limits established in the sediment quality criteria (Cu: 3,860, $\mathrm{Zn}: 2,600, \mathrm{~Pb}: 240, \mathrm{Cd}$ : $240 \mathrm{mg} / \mathrm{kg}$ ) have been reported in the Central Gulf of California (in Santa Rosalía). Cooper mining activities carried out in Santa Rosalía maritime port, since beginning 19th century to the present, are the most important source of heavy metals to the adjacent coastal region (Jonathan et al., 2016). Although isolated cases of inputs of several contaminants are fundamental in explaining the presence of heavy metals in organisms (Zhang et al. 2017; Ali and Khan 2019), the concentrations of Pb and Cd found in $M$. rosacea do not reflect those inputs. However, the concentrations of essential elements such as $\mathrm{Cu}$ (11.6 $\mu \mathrm{g} / \mathrm{g}$ ) reported for $M$. rosacea in the Gulf of California were five times higher than what was reported in Sinaloa for other fish such as L. colorado (Cu: $2.1 \mu \mathrm{g} / \mathrm{g})$.

Organisms that feed mainly on invertebrates, present lower heavy metal levels than those who include a greater proportion of fish in their diet (Escobar-Sánchez et al. 2016; Murillo-Cisneros et al. 2018; Sujitha et al. 2019). M. rosacea is a predator that feeds mainly on the euphausiid Nyctiphanes simplex, which comprises $65 \%$ of the diet, in terms of relative importance (Moreno-Sánchez et al. 2019). Therefore, it should be expected that due to the type of feeding shown by M. rosacea, average heavy metal concentrations would be at the limit (Pb) and/or below the limit (Cd) set by NOM-242-SSA-2009.

Other variables such as size and sex could have an effect on the bioaccumulation of heavy metals (Xia et al., 2019). For example, some elements such as $\mathrm{Hg}$ and $\mathrm{Cd}$ have shown a positive relationship with fish size (Tremain and Adams 2012; Ruelas-Inzunza et al. 2014), where there were greater heavy metal concentrations at greater fish sizes. This was reported for other grouper species (Epinephelus, Mycteroperca, and Cephalopholis) (Tremain and Adams 2012). However, this correlation was not directly proportional with growth (in size, age, or weight) for other heavy metals such as $\mathrm{Pb}, \mathrm{Zn}$, and Cu (García- 
Hernández et al. 2007; Rodrigues et al. 2018; Xia et al. 2019). In this study, there was no evident relationship between total length and the analyzed heavy metals. García-Hernández et al. (2007) showed that the lack of correlation between size and heavy metal concentrations ( $\mathrm{Hg}$, specifically) can be related to different fish species feeding on the same food components.

Nyctiphanes simplex is the main $M$. rosacea prey in Santa Rosalía, however, this grouper can vary the proportion of food items in its diet according to size (Moreno-Sánchez et al. 2019). For example, smallsized grouper (<36 cm; $n$ : 129) fed exclusively on euphausiids ( $N$. simplex), whereas medium-sized fish (>36 cm < $51 \mathrm{~cm} ; n: 99)$ had a mixed diet (invertebrates and fish), and large organisms ( $>51 \mathrm{~cm} ; n: 20)$ consumed a greater proportion of fish (S. sagax, Microlepidotus inornatus, Chromis atrilobata) (MorenoSánchez et al. 2019). As mentioned previously, this would imply that large organisms would present greater heavy metal concentrations (Murillo-Cisneros et al. 2018). However, despite the fact that differences in the diet were reflected in the heavy metal concentrations by size (small, medium, and large), the greatest heavy metal concentrations were recorded in the small and medium-sized organisms (see Table 2) and not in the largest ones. Sujitha et al. (2019) reported that in different crustacean species (e.g., Panulirus interruptus, Penaeus stylirostris) high Zn $(>80 \mu \mathrm{g} / \mathrm{g})$ and $\mathrm{Cu}(>45 \mu \mathrm{g} / \mathrm{g})$ levels occurred, which could explain the presence of greater quantities of those elements in smaller $M$. rosacea.

The occurrence of greater $\mathrm{Cu}$ and $\mathrm{Zn}$ concentrations in small-sized fish could be due to small individuals showing accelerated growth in their first years of life (1 to 3 years), as these elements are metabolically required to carry out necessary physiological processes. Moreover, the elimination rate of toxic metals is more efficient (Rajeshkumar and Li 2018). The size at first maturity of $M$. rosacea has been estimated at $40.7 \mathrm{~cm}$ total length (Pérez-Olivas et al. 2018), which coincides with essential element concentrations being greater in small and medium-sized fish. That is, it should be expected that groupers of this size should have higher heavy metal levels, because $\mathrm{Cu}$ and $\mathrm{Zn}$ are important micronutrients for gonad maturation and are necessary to carry out reproductive events successfully (Jezierska et al. 2009). We suggest that the low $\mathrm{Pb}$ and $\mathrm{Cd}$ levels recorded could be due to more efficient elimination at those sizes (Yi and Zhang 2012), at least in M. rosacea.

The high levels of $\mathrm{Pb}$ and $\mathrm{Cd}$ by the all maturity stages denote increased metabolic activity and high lipid content of the gonads that stimulate rapid accumulation of lipophilic metallic species and are associated to the metallothenin synthesis in the liver (El-Greisy and El-Gamal 2015). The metallothenin proteins binds to metals such $\mathrm{Cd}$ forms a non-toxic complex that is retained in the tissue, resulting in bioaccumulation process. This induction showed that sexual maturity helps to understand the bioavailability of the metals and destoxification of both essential and nonessential metals (Hemmadi 2016).

Compared with what was reported for the El Niño event of 1997 and 1998, the El Niño phenomenon of 2015 was characterized as being the most intense event recorded, with temperatures over $0.76^{\circ}$ higher than the previous record (Pérez-Olivas et al. 2018). Because of this, there was a difference of $2.2^{\circ} \mathrm{C}$ between 2014 and 2015. This difference was reflected in the metal concentrations, with greater 
concentrations in 2015 (Cu: $26 \mu \mathrm{g} / \mathrm{g}$, Zn: $867 \mu \mathrm{g} / \mathrm{g}, \mathrm{Cd}: 0.2 \mu \mathrm{g} / \mathrm{g}, \mathrm{Pb}: 1.5 \mu \mathrm{g} / \mathrm{g}$ ) than in 2014 (Cu: $8.8 \mu \mathrm{g} / \mathrm{g}$, $\mathrm{Zn:} 251 \mu \mathrm{g} / \mathrm{g}, \mathrm{Cd}: 0.08 \mu \mathrm{g} / \mathrm{g}, \mathrm{Pb}: 0.97 \mu \mathrm{g} / \mathrm{g}$ ). These increases in heavy metal concentrations coincide with what was reported by Huerta-Díaz et al. (2014) for the same study area, regarding high heavy metal levels resulting from the mixing and removal of particulate materials in marine sediments, caused by natural phenomena such as hurricanes and storms.

In 2014, hurricane "Odile" impacted Baja California Sur (CONAGUA 2014) in the months categorized as the warm season, coinciding with enrichment in heavy metals during the previously mentioned phenomena. Therefore, the heavy metal enrichment observed in 2015 could be reflecting the impact of that hurricane, which entrained heavy metals through pluvial precipitation and temporary streams flowing into the port. Other activities carried out in Santa Rosalía are not dismissed, where a combination of environmental factors as well as port operations could increase heavy metal concentrations. This increase has also been reported in other heavy metal studies in Northwestern Mexico (Frías-Espericueta et al. 2010; Ruelas-Inzunza et al. 2010; 2014).

Heavy metals are a potential problem in areas close the coast and constitute a human health risk. However, there is currently a discrepancy between recommendations by public health sectors (FAO 2017) and studies that advise reducing the consumption of fish fillet; additionally, the fish consumption by the population is underestimated because the frequency of consumption of fish in coastal communities is often much greater than reported.

In the present study, a consumption of approximately $700 \mathrm{~g}$ does not represent a risk to health. However, the frequency of leopard grouper consumption by the population of Santa Rosalía was greater than what could be recommended here (CONAPESCA 2015). Essential elements such as $\mathrm{Cu}$ and $\mathrm{Zn}$ are required for the correct absorption of vitamins $B_{6}, B_{12}, C$, and $A$, among others, especially in vulnerable groups (children, pregnant women, and lactating women) who require special care to avoid problems of nutrition and embryonic development. According to daily mineral intake values, the $M$. rosacea fillet is highly recommended and beneficial to the population; nutritional values are high and meet levels indicated at worldwide levels (FAO 2017) and are even higher than what has been reported for other fish in Northwestern Mexico (Frías-Espericueta et al. 2010).

The risk factor (THQ) showed no adverse long-term effects, whereas Cd should be considered with caution due to the maximum values obtained. In the present study, we suggest continuous monitoring of Cd levels to evaluate more in depth the THQ and consider later years, because estimated values correspond to 2014 and 2015.

\section{Conclusion}

This is the first study focusing on heavy metals in Myteroperca rosacea. Results on metal concentrations suggest that although the samples of leopard groupers ( $M$. rosacea) come from an area next to mining district "El Boleo" in Santa Rosalía, Mexico, no essential metals ( $\mathrm{Cd}$ and $\mathrm{Pb}$ ) were below the limit set by 
the EPA, FDA, and Mexican norm (NOM-242). Further monitoring is needed for $\mathrm{Pb}$, due to its average was close to the limit set by the Mexican norm. The leopard grouper did not represent adverse effects to the consumer, in contrast, the nutritional benefits are high. Enrichment in heavy metals during warm season may be attributed to impact of natural phenomenon (hurricane) on the study area. Mycteropeca rosacea is still being fished and marketed, therefore it is important to know the ingestion rate of this species in Mexico to understand metal impacts on human populations.

\section{Declarations}

\section{Ethics approval and consent to participate}

Not applicable. This manuscript does not contain any studies with human participants or animals performed by any of the authors.

\section{Consent for publication}

Not applicable

\section{Availability of data and materials}

Not applicable

\section{Competing interest}

The authors declare that they have no conflict of interest.

\section{Funding}

This study is supported by the CONACYT (CONACYT 248708) and Intituto Politécnico Nacional (20150949, 20160319, 20170476, 20181071).

\section{Autor contribution}

Marina S. Irigoyen-Arredondo: Conceptualization, Data analysis, Writing- Original draft preparation. Xchel G. Moreno-Sánchez: Conceptualization, Visualization, Investigation. Ofelia Escobar-Sánchez: Funding acquisition, Project administration, Data curation, Writing- Reviewing and Editing. Martín F. Soto-Jiménez: Data curation, Writing- Reviewing and Editing. Emigdio Marín-Enríquez: Software. L. Andrés AbitiaCárdenas: Conceptualization, Supervision, Investigation, Funding acquisition.

\section{Acknowledgments}

We would like to acknowledge to fisherman from Santa Rosalía, Mexico. Technical assistance was given by Karla Sánchez Osuna. The authors are grateful for economic support obtained from scholarships provided by SIP-IPN $(20150949,20160319,20170476,20181071)$ projects and the research project Problemas Nacionales (CONACYT 248708). MSIA is thankful for economic support derived from 
CONACyT and BEIFI-IPN. XGMS and LAAC are thankful for support obtained from COFAA and EDI scholarships. OES and EME thank CONACYT for financial support through the "CátedrasCONACYT/2137" project. All the authors are grateful to the "Sistema Nacional de Investigadores (SNI)".

\section{References}

1. Ali H, Khan E (2019) Trophic transfer, bioaccumulation, and biomagnification of non-essential hazardous heavy metals and metalloids in food chains/webs Concepts and implications for wildlife and human health. Hum Ecol Risk Assess 25(6):1353-1376.

https://doi.org/10.1080/10807039.2018.1469398

2. Chien LC, Hung TC, Choang KY, Yeh CY, Meng PJ, Shieh MJ, Han BC (2002) Daily intake of TBT, Cu, $\mathrm{Zn}, \mathrm{Cd}$ and As for fishermen in Taiwan. Sci Total Environ 285:177-185. https://doi.org/10.1016/S0048-9697(01)00916-0

3. CONAGUA (2014) Comisión Nacional del Agua. Declaratoria de Desastre Natural por la ocurrencia del Huracan "Odile" del 14 al 15 de septiembre del 2014, en 5 municipios del Estado de Baja California Sur. http://files.conagua.gob.mx/transparencia/PNH2014.pdf

4. CONAPESCA (2014) Comisión Nacional de Pesca y Acuacultura. Anuario Estadística de Pesca. http://www.conapesca.sagarpa.gob.mx/wb/cona/anuario_2014

5. Craig MT, Hastings PA (2007) A molecular phylogeny of the groupers of the subfamily Epinephelinae (Serranidae) with a revised classification of the Epinephelini. Ichth Res 54(1):1-17. https://doi.org/10.1007/s10228-006-0367-x

6. De la O-Burrola VG, De la Rosa-Gutiérrez L, Astorg-Ceja J, Acosta-Martínez Al (2015) El Consumo de Pescados y Mariscos en Baja California. Universidad Autónoma de Baja California. Mexicali, Baja California, México

7. Díaz-Uribe JG, Elorduy-Garay JF, González-Valdovinos MT (2001) Age and growth of the leopard grouper, Mycteroperca rosacea, in the southern Gulf of California, Mexico. Pac Sci 55:171-182. https://doi.org/10.1353/psc.2001.0012

8. El-Greisy ZA, El-Gamal AHA (2015) Experimental studies on the effect of cadmium chloride, zinc acetate, their mixture and the mitigation with vitamin $C$ supplementation on hatchability, size and quality of newly hatched larvae of common carp. Cyprinus carpio Egypt J Aquat Res 41:219-226. https://doi.org/10.1016/j.ejar.2015.03.007

9. Escobar-Sánchez O, Ruelas-Inzunza J, Moreno-Sánchez XG, Romo-Piñera AK, Frías-Espericueta MG (2016) Mercury concentrations in Pacific angel sharks (Squatina californica) and prey fishes from Southern Gulf of California, Mexico. Bull Environ Contam Toxicol 96:15-19. https://doi.org/10.1007/s00128-015-1708-0

10. Erisman B, Craig MT (2018) Mycteroperca rosacea. The IUCN Red List of Threatened Species 2018 : e.T14053A100466656. https://dx.doi.org/10.2305/IUCN.UK.2018-2.RLTS.T14053A100466656.en 
11. Evers DC, Graham RT, Perkins CR, Michener R, Divoll T (2009) Mercury concentrations in the goliath grouper of Belize: an anthropogenic stressor of concern. Endanger Species Res 7(3):249-256. https://doi.org/10.3354/esr00158

12. Food, Organization A (2017) The State of Food Security and Nutrition in the world. In: FAO (ed) cooperation with IFAD (International Fund for Agricultural Development, UNICEF (United Nations Children's Fund), WFP (World Food Programme) and WHO (World Health Organization). Building resilience for peace and food security, Rome

13. Frías-Espericueta MG, Quintero-Alvarez JM, Osuna-López JI, Sanchez-Gaxiola CM, López-López G, Izaguirre-Fierro G, Voltolina D (2010b) Metal contents of four commercial fish species of NW Mexico. Bull Environ Contam Tox 85(3):334-338. https://doi.org/10.1007/s00128-010-0092-z

14. Froese R, Pauly D (2021) FishBase. World Wide Web electronic publication. https://www.fishbase.org (02/2021). Accessed 20 April 2021

15. García-Hernández J, Cadena-Cárdenas L, Betancourt-Lozano M, García-De la Parra LM, García-Rico L, Márquez-Farías F (2007) Total mercury content found in edible tissues of top predator fish from the Gulf of California, Mexico. Toxicol Environ Chem 89(3):507-522. https://doi.org/10.1080/02772240601165594

16. Gu YG, Lin Q, Hong-Hui H, Liang-Gen W, Jia-Jia N, Fei-Yan D (2017) Heavy metals in fish tissues/stomach contents in four marine wild commercially valuable fish species from the western continental shelf of South China Sea. Mar Pollut Bull 114:1125-1129. https://doi.org/10.1016/j.marpolbul.2016.10.040

17. Hemmadi $V$ (2016) Metallothionein - A potential biomarker to assess the metal contamination in marine fishes - A review. Int J Bioassays 5(4):4961-4973

18. Huerta-Díaz MA, Muñoz-Barbosa A, Otero XL, Valdivieso-Ojeda J, Amaro-Franco EC (2014) High variability in geochemical partitioning of iron, manganese and harmful trace metals in sediments of the mining port of Santa Rosalia, Baja California Sur, Mexico. J Geochem Explor 145:51-63. https://doi.org/10.1016/j.gexplo.2014.05.014

19. INEGI (2015) Instituto nacional de estadística y geografía. https://www.beta.inegi.org.mx/areasgeograficas/?ag=03\#

20. Jezierska B, Lugowska K, Witeska M (2009) The effects of heavy metals on embryonic development of fish (a review). Fish Physiol Biochem 35(4):625-640. https://doi.org/10.1007/s10695-008-9284-4

21. Moreno-Sánchez XG, Pérez-Rojo MP, Irigoyen-Arredondo MS, Marín-Enríquez E, Abitia-Cárdenas LA, Escobar-Sánchez $O$ (2019) Feeding habits of the leopard grouper Mycteroperca rosacea (Streets, 1877 ) in the central Gulf of California, BCS, México. Act Icth Pisc 49(1):9-22. https://doi.org/10.3750/AIEP/02321

22. Murillo-Cisneros DA, O'Hara TM, Castellini JM, Sánchez-González A, Elorriaga-Verplancken FR, Marmolejo-Rodríguez AJ, Marín-Enríquez E, Galván-Magaña F (2018) Mercury concentrations in three ray species from the Pacific coast of Baja California Sur, Mexico: Variations by tissue type, sex and length. Mar Pollut Bull 126:77-85. http://dx.doi.org/10.1016/j.marpolbul.2017.10.060 
23. Nikolsky GV (1963) The Ecology of Fishes. Academic Press, New York

24. NOM-242-SSA1-2009. Norma Oficial Mexicana (2009) Bienes y Servicios. Productos de la pesca. Pescados frescos-refrigerados y congelados. Especificaciones sanitarias. Publicación: 10 de febrero del 2011

25. Pal J, Shukla BN, Maurya AK, Verma HO, Pandey G, Amitha A (2018) A review on role of fish in human nutrition with special emphasis to essential fatty acid. Int J Fish Aquat Stud 6(2):427-430

26. Pérez-Olivas A, Irigoyen-Arredondo MS, Moreno-Sánchez XG, Villalejo-Fuerte MT, Abitia-Cárdenas LA, Escobar-Sánchez $O$ (2018) Reproductive biology of the leopard grouper Mycteroperca rosacea (Streets, 1877) in the coastal area of Santa Rosalía, BCS, Mexico. Lat. Am J Aquat Res 46(4):699708. https://doi.org/10.3856/vol46-issue4-fulltext-7

27. Rajeshkumar S, Li X (2018) Bioaccumulation of heavy metals in fish species from the Meiliang Bay, Taihu Lake, China. Toxicol rep 5:288-295. https://doi.org/10.1016/j.toxrep.2018.01.007

28. Rodrigues AM, Antunes P, Paulo L, Pereira ME, Pinto de Andrade L (2018) Metal Contaminants in Largemouth Bass (Micropterus salmoides, Lacépéde, 1802) from Different Origins. Int J Res Agr For $1(5): 8-14$

29. R Core Team (2017) R: a Language and Environment for Statistical Computing, Vienna, Austria. URL http://CRAN.R-project.org/

30. Rehman M, Liu L, Wang Q, Saleem MH, Bashir S, Ullah S, Peng D (2019) Copper environmental toxicology, recent advances, and future outlook: A review. Environ Sci Pollut Res 26(18):1800318016. https://doi.org/10.1007/s11356-019-05073-6

31. Ruelas-Inzunza JR, Escobar-Sánchez O, Páez-Osuna F (2014) Mercury in fish, crustaceans and mollusks from estuarine areas in the Pacific Ocean and Gulf of Mexico under varying human impact. In: Amezcua F and Bellgraph B (eds) Fisheries Management of Mexican and Central American Estuaries, Springer Netherlands, pp 39-49

32. Ruelas-Inzunza J, Páez-Osuna F, García-Flores YD (2010) Essential (Cu) and nonessential (Cd and $\mathrm{Pb}$ ) metals in ichthyofauna from the coasts of Sinaloa state (SE Gulf of California). Environ Monit Assess 162(1-4):251-263. https://doi.org/10.1007/s10661-009-0793-0

33. Shumilin E, Jiménez-Illescas AF, López-López S (2013) Anthropogenic contamination of metals in sediments of the Santa Rosalía Harbor, Baja California Peninsula. Bull Environ Contam Toxicol 90:333-337. https://doi.org/10.1007/s00128-012-0923-1

34. Soto-Jiménez MF, Páez-Osuna F, Scelfo G, Hibdon S, Franks R, Aggarawl J, Flegal AR (2008) Lead pollution in subtropical ecosystems on the SE Gulf of California Coast: A study of concentrations and isotopic composition. Mar Environ Res 66(4):451-458.

https://doi.org/10.1016/j.marenvres.2008.07.009

35. Streets TH (1877) Contributions to the Natural History of the Hawaiian and Fanning Islands and Lower California: Made in Connection with the United States North Pacific Surveying Expedition. US Government Printing Office, pp 1873-1875 (No. 7). 
36. Sujitha SB, Jonathan MP, Aurioles-Gamboa D, Villegas LEC, Bohórquez-Herrera J, HernándezCamacho CJ (2019) Trace elements in marine organisms of Magdalena Bay, Pacific Coast of Mexico: Bioaccumulation in a pristine environment. Environ Geochem Health 41(3):1075-1089. https://doi.org/10.1007/s10653-018-0198-5

37. Thomson DA, Findley LT, Kerstitch AN (2000) Reef fishes of the Sea of Cortez: the rocky-shore fishes of the Gulf of California. University of Texas Press, USA

38. Tremain DM, Adams DH (2012) Mercury in groupers and sea basses from the Gulf of Mexico: Relationships with size, age, and feeding ecology. T Am Fish Soc 141(5):1274-1286. https://doi.org/10.1080/00028487.2012.683232

39. UK EEA (European Environment Agency) (2011) European Union emission inventory report under the UNECE Convention on Long-range Transboundary Air Pollution (LRTAP). Technical Report No 9/2011. Copenhagen

40. US EPA. United States Environmental Protection Agency (2015) Risk-Based Screening Table. https://www.epa.gov/risk/regional-screening-levels-rsls-generic-tables. Accessed 25 May 2020

41. Xia W, Chen L, Deng X, Liang G, Giesy JP, Rao Q, Wen Z, Wu Y, Chen J, Xie P (2019) Spatial and interspecies differences in concentrations of eight trace elements in wild freshwater fishes at different trophic levels from middle and eastern China. Sci Total Environ 672:883-892. https://doi.org/10.1016/j.scitotenv.2019.03.134

42. Xu DM, Yan B, Chen T, Lei C, Lin HZ, Xiao XM (2017) Contaminant characteristics and environmental risk assessment of heavy metals in the paddy soils from lead (Pb)-zinc ( $\mathrm{Zn})$ mining areas in Guangdong Province, South China. Environ Sci Pollut Res 24(31):24387-24399. https://doi.org/10.1007/s11356-017-0052-9

43. Yi YJ, Zhang SH (2012) The relationships between fish heavy metal concentrations and fish size in the upper and middle reach of Yangtze River. Procedia Environ Sci 13:1699-1707. https://doi.org/10.1016/j.proenv.2012.01.163

44. Yi YJ, Yang ZF, Zhang SH (2011) Ecological risk assessment of heavy metals in sediment and human health risk assessment of heavy metals in fishes in the middle and lower reaches of the Yangtze River basin. Environ Pollut 159:2575-2585. https://doi.org/10.1016/j.envpol.2011.06.011

45. Zhang L, Zhu L, Li F, Liu C, Yang Z, Qiu Z, Xiao M (2017) Heavy metals and metalloid distribution in different organs and health risk assessment for edible tissues of fish captured from Honghu Lake. Oncotarget 8(60):101672-101685. https://doi.org/10.18632/oncotarget.21901

\section{Figures}





Figure 1

Geographic location of the port of Santa Rosalía, Baja California Sur, Mexico.

\section{Supplementary Files}

This is a list of supplementary files associated with this preprint. Click to download.

- FigureAbstract.jpg 